\title{
Magnetic transitions and electrical transport in Bi-doped lanthanum strontium manganites
}

\author{
A.M. Ahmed and H.F. Mohamed \\ Physics Department, Faculty of Science, Sohag University, Sohag 82524, Egypt \\ E-mail: fikry_99@yahoo.com \\ Martin Šoka \\ Slovak University of Technology, Faculty of Electrical Engineering and Information Technology \\ Ilkovicova 33, Bratislava 81219, Slovak Republic
}

Received September 2, 2013

\begin{abstract}
The temperature dependence of the electrical resistivity $\rho$, thermoelectric power $S$ and the magnetic susceptibility $\chi$ of $\mathrm{La}_{0.7-} \mathrm{Bi}_{\chi} \mathrm{Sr}_{0.3} \mathrm{MnO}_{3}\left(x=0.05,0.10\right.$, and 0.15 at.\%) manganites were investigated. $\mathrm{La}_{0.7-\chi} \mathrm{Bi}_{\chi} \mathrm{Sr}_{0.3} \mathrm{MnO}_{3}$ crystallizes in a single phase rhombohedral structure with parasitic phase inclusions. With increasing Bi concentration, a systematic decrease in the ferromagnetic transition temperature $\left(T_{C}\right)$, the metal-semiconducting transition temperature $\left(T_{m s 1}\right)$ and also the values of activation energies $E_{\rho}$ and $E_{S}$ from $\rho(T)$ and $S(T)$ were observed. On the other hand, in the high-temperature $\left(T>T_{m s}\right)$ paramagnetic semiconductor regime, the adiabatic small polaron hopping model fit well, thereby indicating that polaron hopping might be responsible for the conduction mechanism. In addition, the thermoelectric power data at low temperatures were analyzed by considering both the magnon and the phonon drag concept, while the high-temperature data were confirmed a small polaron hopping conduction mechanism.
\end{abstract}

PACS: 71.27.+a Strongly correlated electron systems; heavy fermions;

75.30.Hx Magnetic impurity interactions;

75.50.Ee Antiferromagnetics.

Keywords: crystal structure, magnetic properties, electrical conductivity, x-ray diffraction, oxides.

\section{Introduction}

Perovskite-type oxide LAMO $\left(\mathrm{LaAMnO}_{3}\right.$, where $\mathrm{A}$ is a divalent alkaline earth metal ion such as $\mathrm{Sr}^{2+}$ or $\mathrm{Ca}^{2+}$ ) exhibits colossal magnetoresistance (CMR) with a magnetic resistance ratio of more than $100 \%$ [1-4]. In particular, CMR appears near the point of transition from the antiferromagnetic insulator phase to the ferromagnetic metallic phase, and it is closely associated with $\mathrm{Mn}$ in the LAMO specimen having a large spin polarization based on strong Hund's rule coupling [5]. In this case, the electrical conduction characteristics depend on whether a conduction electron enters an electron orbit in terms of the Jahn-Teller (JT) strain which accompanies the symmetry of the crystal structure [6]. It is known that by doping the bismuth to the system LAMO, the electrical resistivity and magneto-optical effect change [7], but the details are not clear. In order to reveal the mechanism of magneto-transport, Righi et al. [8] have investigated the Bi-doping effects on the structural, transport and magnetic properties of $\mathrm{La}_{0.7-} \mathrm{Bi}_{\chi} \mathrm{Sr}_{0.3} \mathrm{MnO}_{3}$, and have found that the dopant $\mathrm{Bi}$ cause structure change and decreases the $T_{m s}$. However, interpretation of the temperature dependence of the thermoelectric power (TEP) $S(T)$ for transition metal oxide is rarely reported [9-11] due to the complexity of elucidating the $S(T)$ apart from the diffusion TEP or temperature-independent TEP.

As we know, there are many different properties in $\mathrm{La}_{1-x} \mathrm{Ca}_{\chi} \mathrm{MnO}_{3}$ and $\mathrm{La}_{1-\chi} \mathrm{Sr}_{x} \mathrm{MnO}_{3}$, such as metal-insulator transition temperature at optimal doping and the critical doping concentration for the presence of ferromagnetizm [12,13]. So, we have investigated systematically the Bi-doping effect on the magnetic and electrical properties in $\mathrm{La}_{0.7-x} \mathrm{Bi}_{x} \mathrm{Sr}_{0.3} \mathrm{MnO}_{3}$ with the expectation that it will provide new insight and interesting physics.

\section{Experimental}

All samples reported in the present study were synthesized by a standard solid-state reaction procedure. Stoichiometric compositions of $\mathrm{La}_{0.7-x} \mathrm{Bi}_{x} \mathrm{Sr}_{0.3} \mathrm{MnO}_{3}$ ( $x=0.05$, 0.10 , and 0.15 at.\%) were prepared by mixing equimolar amounts of $\mathrm{La}_{2} \mathrm{O}_{3}, \mathrm{Bi}_{2} \mathrm{O}_{3}, \mathrm{SrO}$, and $\mathrm{MnCO}_{3}$, respectively 
(all having greater than $99.99 \%$ purity). The powders of these oxides and the carbonate were mixed and were finely ground in an electric grinder for $30 \mathrm{~min}$. After grinding, the powders were pressed into pellets with a pressure of 2 ton/ $\mathrm{cm}^{2}$ and calcined at $1173 \mathrm{~K}$ for $8 \mathrm{~h}$ followed by cooling to room temperature, they were reground and again pressed into pellets with a pressure of $7 \mathrm{ton} / \mathrm{cm}^{2}$ and subsequently calcined at $1373 \mathrm{~K}$ for $6 \mathrm{~h}$ [14]. Samples were checked by $\mathrm{x}$-ray powder diffraction analysis indicating the presence of a unique phase with perovskite-type structure. Resistivity measurements were performed in a commercial variable temperature liquid nitrogen cryostat. The resistivity was measured as a function of temperature using the standard four-probe method and air-drying conducting silver paste as in previous works $[14,15]$. The thermoelectric power measurements were carried using the sample two-heater method with copper electrodes see Refs. 15-17. The magnetic susceptibility measurement was performed, from room temperature to $700 \mathrm{~K}$, using the Kappa Bridge KLY-2 with operating frequency $920 \mathrm{~Hz}$.

\section{Results and discussion}

The x-ray diffraction patterns of the $\mathrm{La}_{0.7-x} \mathrm{Bi}_{\chi} \mathrm{Sr}_{0.3} \mathrm{MnO}_{3}$ $(x=0.05,0.10$, and 0.15 at.\%) show that the systematic substitution of La by Bi does not produce relevant effect on them. In general, all the peaks for samples satisfy the La-Sr-Mn-O phase. In addition, some weak impurity peaks from $\mathrm{SrMnO}$, $\mathrm{BiSrMnO}$, and $\mathrm{Bi}_{2} \mathrm{O}_{3}$ phases were found [14]. The crystal structure for the compositions $\mathrm{La}_{0.7-\chi} \mathrm{Bi}_{x} \mathrm{Sr}_{0.3} \mathrm{MnO}_{y}$ was found to be rhombohedral structure [18-20]. Lattice parameters and cell volume were calculated and tabulated in Table 1.

As seen in Table 1 the lattice parameter $a$ and unit-cell volume slight increase with increasing $\mathrm{Bi}$ concentration, while parameter $c$ slight decreases with $x$. This almost perfect match can be explained considering the similar dimension of the two cations $\mathrm{La}^{3+}$ (ionic radius $r=1.032 \AA$ ) and $\mathrm{Bi}^{3+}$ (ionic radius $r=1.030 \AA$ ) [21].

Figure 1 shows the variation of resistivity with temperature for $\mathrm{La}_{0.7-} \mathrm{Bi}_{x} \mathrm{Sr}_{0.3} \mathrm{MnO}_{3}$. Obviously, the resistivity increases with $\mathrm{Bi}$ doping. We expect that when the Bicontent increases not only the La-content decreases but also the charge carrier density [22] which leads to a reduction of the double exchange which is proportional to bandwidth. Therefore, the $\mathrm{La} / \mathrm{Bi}$ configuration plays a prominent role in controlling the resistivity. Consistently, the figure shows that the transition temperature $\left(T_{m s}\right)$ for

Table 1. The lattice parameters and cell volume $(V)$ with concentration of the $\mathrm{La}_{0.7-} \mathrm{Bi}_{x} \mathrm{Sr}_{0.3} \mathrm{MnO}_{3}$

\begin{tabular}{c|c|c|c}
\hline \hline \multirow{2}{*}{ Parameter } & \multicolumn{3}{|c}{ Bi content, at.\% } \\
\cline { 2 - 4 } & 0.05 & 0.10 & 0.15 \\
\hline \hline$a, \AA$ & 6.043 & 6.050 & 6.052 \\
$c, \AA$ & 7.760 & 7.760 & 7.748 \\
$V, \AA^{3}$ & 245.41 & 245.98 & 245.76 \\
\hline \hline
\end{tabular}

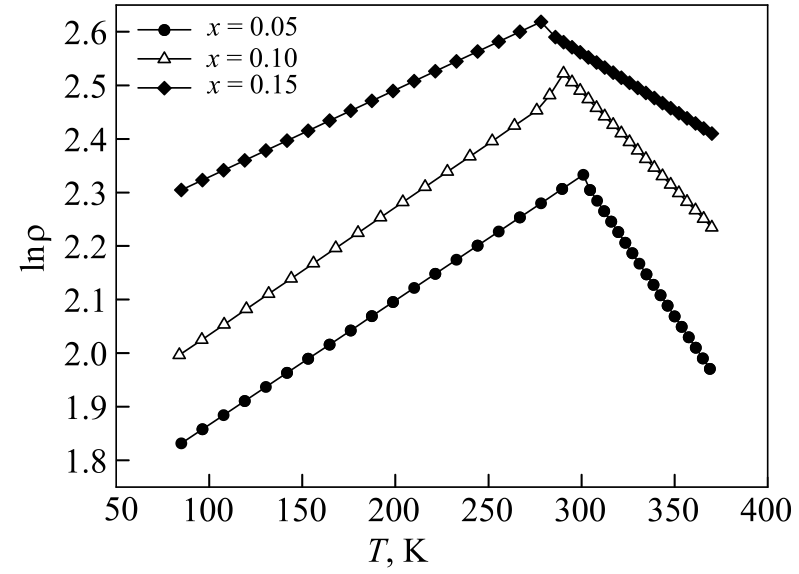

Fig. 1. In $\rho$ versus temperature for $\mathrm{La}_{0.7-} \mathrm{Bi}_{x} \mathrm{Sr}_{0.3} \mathrm{MnO}_{3}$.

$\mathrm{La}_{0.7-} \mathrm{Bi}_{\chi} \mathrm{Sr}_{0.3} \mathrm{MnO}_{3}$ decreases with increasing Bi content. These compounds have a distinct metallic phase below the transition temperature $\left(T_{m s 1}\right)$ and above this temperature they become semiconducting (S). In addition both the change in carrier concentration and $T_{m s} / T_{C}$ with Bi content can be interpreted as arising from the rather covalent character of the $\mathrm{Bi}-\mathrm{O}$ bonds (which are shorter than the $\mathrm{La}-\mathrm{O}$ due to the covalent character of the former). That, in turn, contributes to the localization of the oxygen electrons coupling the $\mathrm{Mn}^{3+/ 4+}$ ions, and could explain the increase of the antiferromagnetic interactions and the decrease of the metallic character for the Bi-substituted compounds, ending up with the totally $\mathrm{AF}$ and insulating $\mathrm{Bi}-\mathrm{Sr}-\mathrm{MnO}_{3}$.

The resistivities data above $T_{m s 1}$ (PM-S region) are analyzed in view of small polaron hopping (SPH) are generally used where the transition temperatures of our composites are high temperature. The data are fitted well with the SPH model of Mott [23] viz.,

$$
\rho / T=\rho_{\alpha} \exp \left(E_{\rho} / k_{B} T\right)
$$

where $E_{\rho}$ is the energy equal to $W_{D} / 2+W_{H}$; for $T>T_{m s 1}$ (where $W_{H}$ is the polaron hopping energy and $W_{D}$ is the disorder energy). $E_{\rho}$ and $\rho_{\alpha}$ calculated and tabulated in Table 2. Indeed, as a result of the fit, the adiabatic SPH model is used in the present investigation. As in Table 2 both $E_{\rho}$ and $\rho_{\alpha}$ decrease with increasing $\mathrm{Bi}$ content. This behavior is explained by considering that increasing $x$ causes charge delocalization (due to decrease of small

Table 2. The variation of $E_{\rho}(\mathrm{meV}), \rho_{\alpha}(\Omega \cdot \mathrm{cm}), E_{S}(\mathrm{meV}), B$ and $W_{H}(\mathrm{meV})$ with concentration for $T>T_{m s}$

\begin{tabular}{c|c|c|c}
\hline \hline \multirow{2}{*}{ Parameter } & \multicolumn{3}{|c}{ Bi content, at.\% } \\
\cline { 2 - 4 } & 0.05 & 0.10 & 0.15 \\
\hline \hline$\rho_{\alpha}$ & 6.35 & 6.33 & 6.31 \\
$E_{\rho}$ & 77.50 & 76.60 & 76.24 \\
$E_{S}$ & 11.90 & 9.40 & 7.50 \\
$B$ & 0.035 & 0.022 & 0.022 \\
$W_{H}$ & 65.40 & 67.50 & 68.74 \\
\hline \hline
\end{tabular}


polaron coupling constant or el-ph interaction constant) in the system and thereby the energy required to liberate a free carrier is reduced.

To discuss the nature of the conduction mechanism below $T_{m s 1}$ (FM-M region), the resistivity data are fitted with three empirical equations derived by different previous work [24-26]:

$$
\begin{gathered}
\rho=\rho_{0}+\rho_{2} T^{2}, \\
\rho=\rho_{0}+\rho_{2.5} T^{2.5}, \\
\rho=\rho_{0}+\rho_{2} T^{2}+\rho_{4.5} T^{4.5},
\end{gathered}
$$

where $\rho_{0}$ represents the resistivity due to grain boundary effects. $\rho_{2} T^{2}$ in term in Eqs. (2) and (4) indicates the resistivity due to electron-electron scattering process and is generally dominant up to $100 \mathrm{~K}$. On the other hand, the term $\rho_{2.5} T^{2.5}$ represents the resistivity due to electronmagnon scattering process in ferromagnetic phase. Finally, the term $\rho_{4.5} T^{4.5}$ indicates the resistivity due to electronmagnon scattering process in ferromagnetic region, which may be likely to arise due to spin-wave scattering process.

Our data of the metallic (ferromagnetic) part of the temperature-dependent resistivity $(\rho)$ curve (below $T_{m s}$ ) fits well with Eq. (4) $\left(R^{2}>99.9 \%\right)$. Indicating the importance of grain/domain boundary effects and electronmagnon scattering processes in the conduction of our composites. As in Table 3 the values of $\rho_{0}>\rho_{2}>\rho_{4.5}$, this means that both grain boundaries and electron-electron scattering process play a role besides an electron-magnon scattering process in conduction mechanism. The last term $\rho_{4.5}$ is also found to decrease with increasing $\mathrm{Bi}$ content; the observed behavior may be due to partial alignment of the spins which results in lowering their fluctuations [27].

The temperature dependence of magnetic susceptibility $(\chi)$ were measured with a magnetic field of $300 \mathrm{~A} / \mathrm{m}$. Figure 2 shows the $\chi-\mathrm{T}$ curves for $\mathrm{La}_{0.7-x} \mathrm{Bi}_{\chi} \mathrm{Sr}_{0.3} \mathrm{MnO}_{3}$ samples ( $x=0.05,0.10$, and 0.15 at.\%), demonstrating the presence of clear FM transitions, while the value of magnetic susceptibility decreases with increasing the doping of $\mathrm{Bi}$ for these samples. This phenomenon can be interpreted as the increased bending of the $\mathrm{Mn}-\mathrm{O}-\mathrm{Mn}$ bond with decreasing average A-site ionic radius $\left\langle r_{\mathrm{A}}\right\rangle$ due to the partial substitution of smaller $\mathrm{Bi}^{3+}$ ions for a bit larger $\mathrm{La}^{3+}$ ions. This substitution causes the narrowing of the bandwidth and the decreasing of the mobility of $e_{g}$ electron resulting in the weakness of DE interaction magnetism [28] (this confirm the $\rho(T)$ data). Based on these results, ferro- to paramagnetic transition temperatures $\left(T_{C}\right)$ were determined from the inflection point of $d \chi / d T$. It is clear from the values of $T_{C}$ values are also following the same trend as those of $T_{m s}$.

Figure 3 shows the dependence of Seebeck coefficient (S) on the temperature. The TEP of these samples, depicted in Fig. 3, is positive at low temperatures, suggesting hole conduction, but becomes negative at high temperatures $(T>300 \mathrm{~K})$. The transition from metallic to semiconducting behavior $\left(T_{m s 2}\right)$ is clearly seen in the figure. Below the $T_{m s 2}$, the value of $S$ increases with increasing Bi doping, above the transition this is also true except for $x=0.15$ at.\%. In addition, above the transition $S$ decreases rapidly. When the resistivity is thermally activated, the thermopower may also be expected to show semiconducting-like behavior. The sign change in $S$ at high temperatures confirms that the coexistence of two types of carriers. The negative $S$ at high temperature is attributed to the electrons which are excited from the valence band (VB) into the conduction band (CB). Because of the higher mobility of electrons within the $\mathrm{CB}, S$ is negative. At low temperatures, the electrons in the VB band are excited into the impurity band which generates hole-like carriers, which is responsible for a positive $S$ [29]. The magnitude of $S$ increases with increasing Bidoping except in the case of $x=0.15$ above $T_{m s 2}$, and the observed behavior due to the fact that for every ion of $\mathrm{Bi}$ doping, double the hole centers, which are localized and causes narrowing of $e_{g}$ band, this have been confirmed by $\chi(T)$ and $\rho(T)$ measurements.

As in many previous work $[30,31]$ that phonon drag $\left(S_{g}\right)$ and magnon drag $\left(S_{m}\right)$ contributions to the diffusion $\left(S_{d}\right)$ in the low-temperature region. In the low-temperature FM-M region, a magnon drag effect is produced due to the presence of electron-magnon scattering, while the phonon drag is due to electron-phonon scattering. In general, we can analyzed $S-T$ relation as (note that $n_{\mathrm{ph}} \sim T^{3}, n_{\text {mag }} \sim T^{3 / 2}$ ):

$$
S=S_{0}+S_{3 / 2} T^{3 / 2}+S_{4} T^{4}
$$

where $S_{0}$ is a constant and accounts the low-temperature variation of thermo-power. The second term $S_{3 / 2} T_{3 / 2}$ is attributed to the magnon scattering process, while the origin of the last term $S_{4} T^{4}$ is related to the spin-wave fluctuations in the FM-M region [30]. We fitted our data using

Table 3. The resistivity data, fitted with empirical equations (2)-(4), due to different scattering process

\begin{tabular}{c|c|c|c|c|c|c}
\hline \hline $\begin{array}{c}\text { Sample } \\
\text { code }\end{array}$ & $\begin{array}{c}\rho= \\
\rho_{0}+\rho_{2} T^{2}\end{array}$ & $\begin{array}{c}\rho= \\
\rho_{0}+\rho_{2.5} T^{2.5}\end{array}$ & $\begin{array}{c}\rho=\rho_{0}+ \\
+\rho_{2} T^{2}+\rho_{4.5} T^{4.5}\end{array}$ & $\rho_{0}, \Omega \cdot \mathrm{cm}$ & $\rho_{2}, 10^{-5} \Omega \cdot \mathrm{cm} / \mathrm{K}^{2}$ & $\rho_{4.5,10^{-11} \Omega \cdot \mathrm{cm} / \mathrm{K}^{4.5}}$ \\
\hline \hline 0.05 & 0.9801 & 0.9602 & 0.9977 & 5.8214 & 6.6775 & 1.3144 \\
0.10 & 0.9921 & 0.9876 & 0.9921 & 7.2756 & 5.7660 & 0.0525 \\
0.15 & 0.9690 & 0.9466 & 0.9950 & 9.4549 & 7.9799 & 2.1155 \\
\hline \hline
\end{tabular}




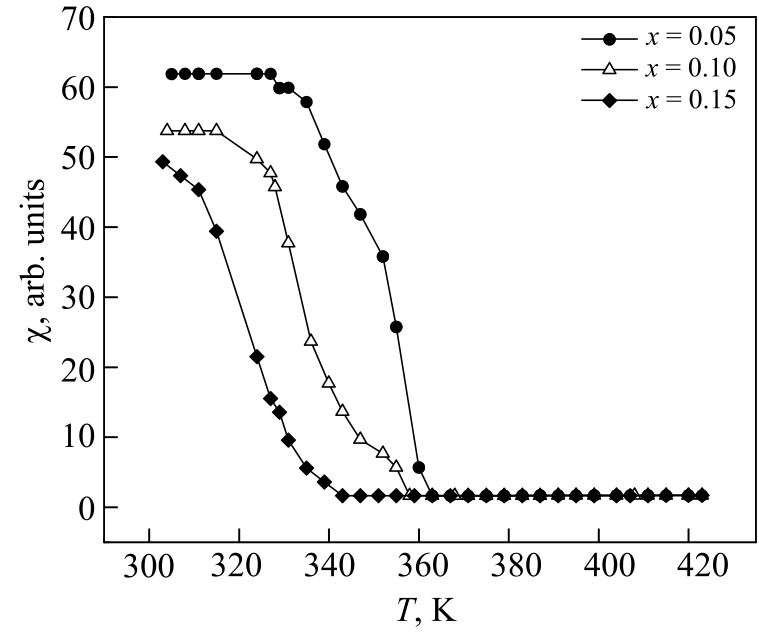

Fig. 2. Temperature dependences of susceptibility for samples $\mathrm{La}_{0.7-\chi} \mathrm{Bi}_{\chi} \mathrm{Sr}_{0.3} \mathrm{MnO}_{3}$.

Eq. (5) and we found that it fit well only for a short range of low temperature. Therefore, we refit our data using the modified Eq. (6), which modified by adding two more terms, honon drag and diffusion drag and the resulting equation is given by [31]

$$
S=S_{0}+S_{1} T+S_{3 / 2} T^{3 / 2}+S_{3} T^{3}+S_{4} T^{4},
$$

where the term $S_{1} T$ and $S_{3} T^{3}$ represent to the diffusion and the phonon drag contribution to the TEP, respectively. The lines in Fig. 4, indicate that Eq. (6) is in good agreement with the experimental results of magnon contribution from 83 up to 313, 273, and $263 \mathrm{~K}$ high of samples with $\mathrm{Bi}$ content $x=0.05,0.10$, and 0.15 at.\%, respectively. But for the contribution of phonon is in good agreement with experimental results from 163-273, 103-253, and 113-263 K of samples with $x=0.05,0.10$, and 0.15 at.\%, respectively (Fig. 4(b)). It follows the linear dependence of $T^{3}$ through a broad temperature regime and becomes zero at $T=0 \mathrm{~K}$. This behavior reflects that the phonon drag effect disappears because the lattice is frozen at $T=0 \mathrm{~K}$. Here, it devi-

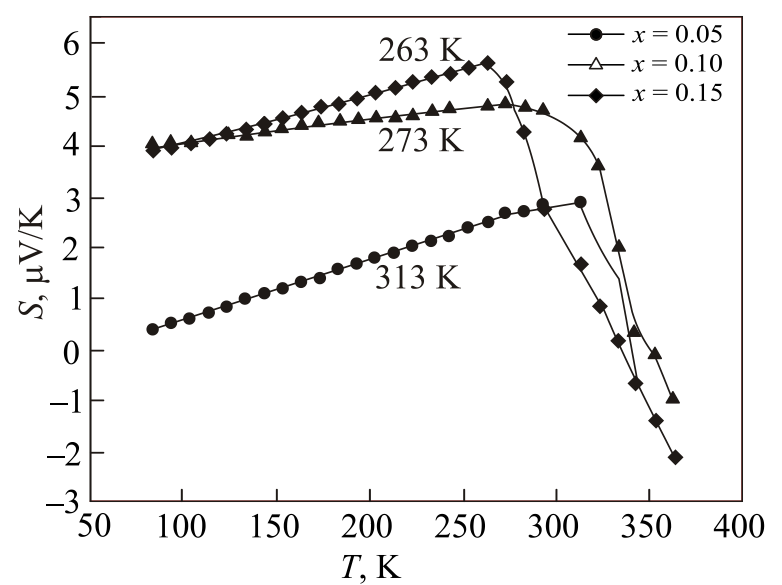

Fig. 3. Temperature dependences of thermoelectric power for $\mathrm{La}_{0.7-\chi} \mathrm{Bi}_{\chi} \mathrm{Sr}_{0.3} \mathrm{MnO}_{3}$.
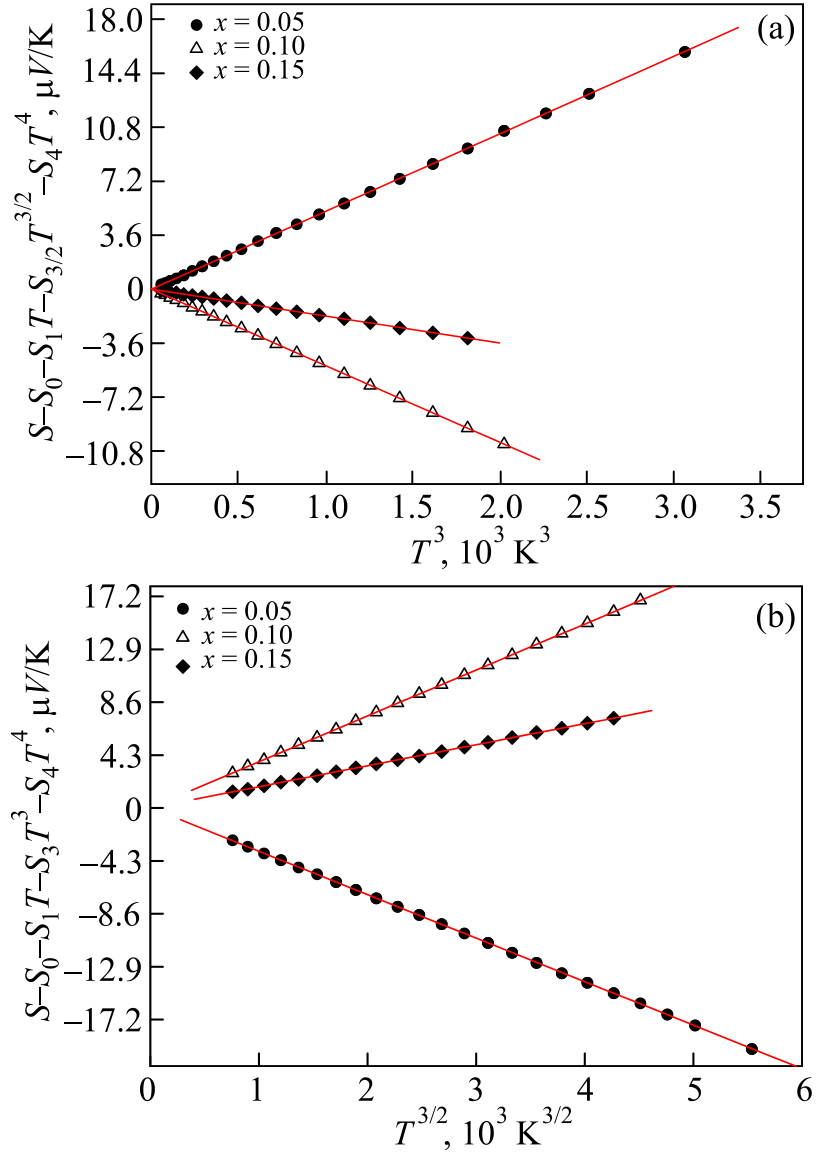

Fig. 4. (Color online) Variations of phonon drag component with $T^{3}$ (a) and magnon drag component with $T^{3 / 2}$ (b). The red lines represent the deviation of linear fit to experimental curve.

ates from the $T^{3}$-dependence below $100 \mathrm{~K}$. The magnon drag component shows $T^{3 / 2}$-behavior in several regimes, especially below $173 \mathrm{~K}$. This indicates that the dominant contribution of TEP in low temperature due to magnon drag effect.

The charge carriers in the semiconductor region are not it inerrant and the transport properties are governed by thermally activated carriers because the effect of JT distortions in manganites results in strong electron-phonon coupling and hence the formation of polarons. Therefore, the thermoelectric power data of the present samples in semiconductor regime are fitted to Mott's polaron hopping equation,

$$
S= \pm k_{B} / e\left(\Delta E_{S} / k_{B} T+B\right)
$$

where $k_{B}$ is the Boltzmann constant, $e$ is the electronic charge, $E_{S}$ is the activation energy obtained from thermoelectric power data, and $B$ is a constant. In Eq. (7), $B<1$ implies the applicability of small polaron hopping model, whereas $B<2$ indicates the large polaron hopping. From the slope and the intercept of $S$ versus $1 / T$ curves (Fig. 5), we obtain the values of activation energy $E_{S}$ and the constant $B$ (Table 2). The estimated values of $B$ indicated $B<1$ for three samples. Therefore, the small polaron hopping conduc- 

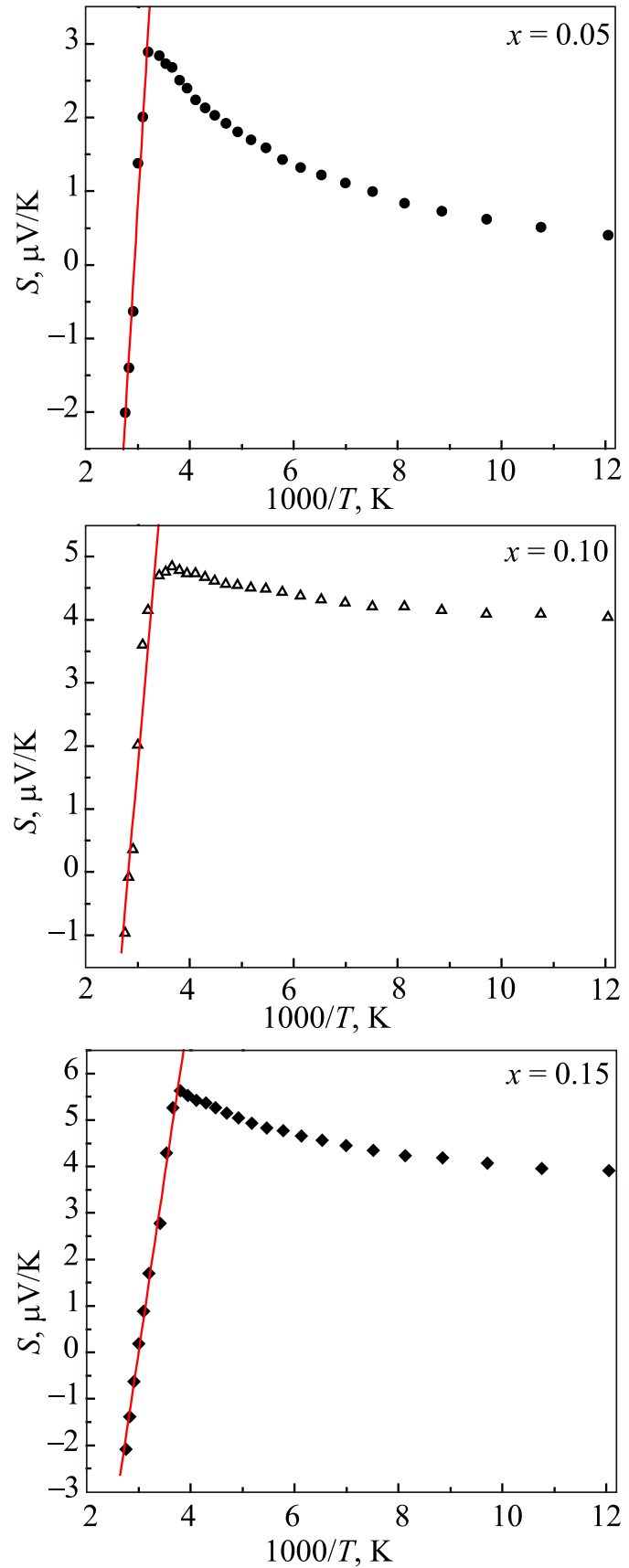

Fig. 5. (Color online) Variations of $S$ vs $1 / T$ for samples $\mathrm{La}_{0.7-}$ ${ }_{x} \mathrm{Bi}_{x} \mathrm{Sr}_{0.3} \mathrm{MnO}_{3}$. The red lines represent the best fit to SPH model.

tion mechanism is also strongly supported by the high temperature $\left(T>T_{m s}\right)$ TEP data. From conductivity data also we have approved of the possibility of the formation of small polaron hopping conduction mechanism. Using the activation energy values from $\rho(T)$ plots $E_{\rho}$ and those from $S(T)$ plots $E_{S}$, the polaron hopping energy values of all the samples have been calculated using the relation, $W_{H}=E_{\rho}-E_{S}$, and are given in Table 2. The $E_{\rho}$ values are found to be higher than those of $E_{S}$. Such a large difference in the activation energy is confirm also the applicability of the SPH model in the semiconducting region [30].

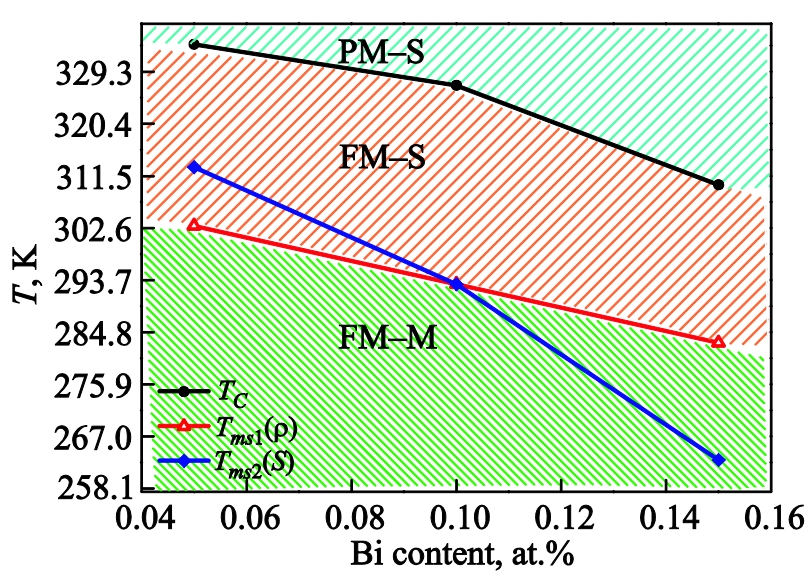

Fig. 6. (Color online) The phase diagram of rhombohedral structure $\mathrm{La}_{0.7-x} \mathrm{Bi}_{x} \mathrm{Sr}_{0.3} \mathrm{MnO}_{3}(x=0.05,0.10,0.15$ at.\%) system.

The Curie temperature $T_{C}$ and the metal-semiconducting transition temperatures $T_{m s 1}$ and $T_{m s 2}$ were deduced from the derivatives of the magnetic susceptibility curves $\rho(T)$ and $S(T)$, respectively. The evolution of $T_{m s 1}$ of $\rho(T)$ and $T_{m s 2}$ of $S(T)$ and $T_{C}$ are shown in Fig. 6. This figure shows the phase diagram of rhombohedral structure $\mathrm{La}_{0.7-x} \mathrm{Bi}_{x} \mathrm{Sr}_{0.3} \mathrm{MnO}_{3}(x=0.05,0.10,0.15$ at.\%) system, where the FM-M phase underlie $T_{m s 1}$ (red line), the FM-S phase lie between $T_{m s 1}$ (red line) and $T_{C}$ (black line), finally, PM-S phase lie above $T_{C}$ (black line).

One interesting feature concerns the value of both $T_{m s 1}$ ( $\rho(T))$ and $T_{m s 2}(S(T)$ ) which is often smaller than the corresponding value of $T_{C}$ (about $40 \mathrm{~K}$ ). These transition temperatures decrease as $x$ increases, as expected. Therefore, we can predict the composition which should lead to the maximum magneto-resistance at the room temperature [32] which is more suitable for applications.

\section{Conclusion}

In conclusion, temperature-dependent (360-80 K) electrical conductivity and thermopower measurements of the Bi-doped $\mathrm{La}_{0.7-x} \mathrm{Bi}_{X} \mathrm{Sr}_{0.3} \mathrm{MnO}_{3}(x=0.05,0.10,0.15$ at.\%) system have revealed metal-semiconduting transitions. In other side, the magnetic properties have showed FM-PM transtion between 310 and $334 \mathrm{~K}$. The high-temperature conductivity data can be successfully fitted with the small polaron-hopping conduction theory like that of usual oxide semiconductors.

The data of Seebeck coefficient supports the small-polaron hopping transport mechanism. Also, the large difference between $E_{\rho}$ and $E_{S}$ provides evidence of small polaron transport mechanism in the high-temperature PM region.

The metallic state below $T_{m s}$ has been considered in terms of the electron-magnon or electron-phonon scattering process depending on the ambient temperature. From the high difference between the values of $T_{m s}$ and $T_{C}$, we predict the maximum magnetoresistance is at room temperature. 


\section{Acknowledgments}

The authors would like to thank Prof. Dr. Marcel Miglierini and Dr. Marius Pavlovic for they help in susceptibility measurements in Slovak University of Technology, Faculty of Electrical Engineering and Information Technology.

1. Y. Tokura, A. Urushibara, Y. Moritomi, T. Arima, A. Asamitsu, G. Kido, and N. Furukawa, J. Phys. Soc. Jpn. 63, 3931 (1994).

2. Takashi Ogawa, Adarsh Sandhu, Masafumi Chiba, Hiromasa Takeuchi, and Yoshiharu Koizumi, J. Magn. Magn. Mater. 290, 933 (2005).

3. A.M. Ahmed, A. Kattwinkel, K. Bärner, C.P. Yang, J.R. Sun, G.H. Rap, H. Schicketanz, P. Terieff, and I.V. Medvedeva, J. Magn. Magn. Mater. 242, 719 (2002).

4. Elena Rezlescu, Corneliu Doroftei, Paul Dorin Popa, and Nicolae Rezlescu, J. Magn. Magn. Mater. 320, 796 (2008).

5. S. Takemoto, R. Takumi, H. Takeuchi, and Y. Koizumi, J. Soc. Powder Metall. 48, 1107 (2001).

6. L.E. Gontchar, A.E. Nikiforov, and S.E. Popov, J. Magn. Magn. Mater. 22, 175 (2001).

7. T.J.A. Popma and M.G.J. Kamminga, Solid State Commun. 17, 1073 (1975).

8. L. Righi, J. Gutiérrez, and J.M. Barandiarán, J. Phys.: Condens. Matter 11, 2831 (1999).

9. W.H. Jung, J. Korean Ceram. Soc. 40, 849 (2003).

10. P. Mandal, Phys. Rev. B 61, 14675 (2000).

11. S. Bhattacharya, A. Banerjee, S. Pal, R.K. Mukherjee, and B.K. Chaudhuri, J. Appl. Phys. 93, 356 (2003).

12. A.P. Ramirez, S.W. Cheong, and P. Schiffer, J. Jpn. Phys. 81, 5337 (1997).

13. R. Maezono, S. Ishihara, and N. Nagaosa, Phys. Rev. B 58, 11583 (1998).

14. A.M. Ahmed, Physica B 352, 330 (2004).

15. A.M. Ahmed, A.K. Diab, and H.F. Mohamed, J. Supercond. Nov. Magn. 24, 597 (2011).
16. A.M. Ahmed, S.A. Saleh, E.M.M. Ibrahim, and H.F. Mohamed, J. Magn. Magn. Mater. 301, 452 (2006).

17. A.M. Ahmed, S.A. Saleh, E.M.M. Ibrahim, E. Bontempib, and H.F. Mohamed J. Magn. Magn. Mater. 320, L43 (2008).

18. X.J. Fan, J.H. Zhang, X.G. Li, W.B. Wu, J.Y. Wan, T.J. Lee, and H.C. Ku, J. Phys.: Condens. Matter 11, 3141 (1999).

19. S. Uhlenbruck, B. Büchner, R. Gross, A. Freimuth, A. Maria de Leon Guevara, and A. Rvecolevschi, Phys. Rev. B 57, R5571 (1998).

20. A. Pimenov, M. Biberacher, D. Ivannikov, A. Loidl, V.Yu. Ivanov, A.A. Mukhin, and A.M. Balbashov, Phys. Rev. B 62, 5685 (2000).

21. G. Srinivasan, R.M. Savage, V. Suresh Babu, and M.S. Seehra, J. Magn. Magn. Mater. 168, 1 (1997).

22. G.H. Rao, J.R. Sun, A. Kattwinkel, L. Haupt, K. Bärner, E. Schmitt, and E. Gmelinn, Physica B 269, 379 (1999).

23. N.F. Mott and E.A. Davis, in Electronics Process in NonCrystalline Materials, Clarendon, Oxford (1971).

24. A. Banerjee, S. Pal, and B.K. Chaudhuri, J. Chem. Phys. 115, 1550 (2001).

25. L. Pi, L. Zhang, and Y. Zhang, Phys. Rev. B 61, 8917 (2000).

26. G. Jeffrey Snyder, R. Hiskes, S. Dicarolis, M.R. Beasley, and T.H. Geballe, Phys. Rev. B 53, 14434 (1996).

27. V. Ravindranath, M.S. Ramachandra Rao, G. Rangar-ajan, Yafeng Lu, J. Klein, R. Klingeler, S. Uhlenbruck, B. Buchner, and R. Gross, Phys. Rev. B 63, 184434 (2001).

28. J.B. Torrance, P. Lacorre, and A.I. Nazzal, Phys. Rev. B 45 , 8209 (1992).

29. J. Yang, Y.P. Sun, W.H. Song, and Y.P. Lee, J. Appl. Phys. 100, 123701 (2006).

30. S. Battacharya, S. Pal, A. Banerjee, H.D. Yang, and B.K. Chaudhuri, J. Chem. Phys. 119, 3972 (2003).

31. B.H. Kim, J.S. Kim, T.H. Park, D.S. Le, and Y.W. Park, J. Appl. Phys. 103, 113717 (2008).

32. G. Srinivasan and D. Hanna, Appl. Phys. Lett. 79, 641 (2001).

33. 